\title{
Zur Kenntnis des Zuckerabbaus beim Diabetes mellitus.
}

Von

\author{
Privatdozent Dr. W. Stepp. \\ (Aus der medizinischen Klinik zu Gießen, Professor Dr. V oit.) \\ (Der Redaktion zugegangen am 8. April 1916.)
}

Die alte Streitfrage, ob die Hyperglykämie beim Diabetes mellitus auf Störungen der Zuckerverwertung oder auf zu reichlicher Zuckerbildung beruht, hat bis heute eine endgültige Entscheidung nicht erfahren. Solange wir nicht den Zuckerabbau beim Gesunden in allen seinen Phasen übersehen können, erscheinen Versuche, in den Kohlenhydratstoffwechsel beim Diabetiker einzudringen, nicht sehr aussichtsvoll. Dagegen wird es möglich sein, gewisse Richtlinien für weitere Forschungen zu bekommen, wenn man sich Aufschluß über die Höhe des Kohlenst offs im enteiweißten Blut verschafft. Die Kohlenhydrate mit ihrem hohen Kohlenstoffgehalt müssen stärkere Schwankungen in deutlichen Ausschlägen des "Kohlenstoffspiegels» anzeigen. Man vergegenwärtigè sich nun, daß beim Diabetes der Blutzucker bis über das 10 fache der Norm ansteigen kann! Es werden hier große Ausschläge in den Kohlenstoffwerten des Blutes zu erwarten sein. Der Traubenzucker enthält $40 \%$ Kohlenstoff, somit entsprechen dem normalen Blutzuckerwert von etwa $0,1 \% 40 \mathrm{mg}$ Kohlenstoff. Steigt der Blutzucker beim Diabetes auf das Mehrfache, so wird die auf ihn entfallende Kohlenstoffmenge für jede Steigerung um 0,1\% mit $40 \mathrm{mg}$ in die Höhe gehen. Nun bildet aber der auf den Blutzucker treffende Kohlenstoffwert nur einen Teil des im enteiweißten Blut vorhandenen Kohlenstoffs, zum großen Teil gehört er unbekannten Verbindungen an. Vergleichende Bestimmungen 
des Blutzuckers und des Kohlenstoffs im enteiweiBten Blut von Diabetes-Kranken werden somit gestatten, das Verhältnis von Zuckerkohlenstoff zum Gesamtkohlenstoff ${ }^{1}$ ) und etwa hierin eintretende Änderungen klar zu übersehen. Vielleicht lassen sich hierbei wichtige Aufschlüsse über den Kohlenhydratabbau gewinnen.

Ich habe solche Untersuchungen ausgeführt und dabei mich der im Laboratorium von Franz Hofmeister für die Ermittelung des sogenannten a Restkohlenstoffs, benutzten EnteiweiBung mit Phosphorwolframsäure bedient. ${ }^{2}$ ) Diese Methode hatte für meine Zwecke erstlich den Vorteil, daß nicht nur sämtliche koagulablen Eiweißkörper einschließlich des Serummukoids, sondern auch nicht eiweißartige Körper, wie die Harnsäure und die Hauptmenge der übrigen Purine, ferner höhere Fettsäuren und so gut wie alle im Blut kolloidal suspendierten Körper entfernt werden.

Hierfür habe ich bezüglich des Cholesterins und der Phosphatide den direkten Nachweis erbracht. Das Filtrat der Fällung von ca. $100 \mathrm{ccm}$ Hundeblut wurde mit Baryumcarbonat neutralisiert und zur Trockne gebracht. Der Rückstand wurde pulverisiert und mit kochendem Alkohol extrahiert, ein Teil des alkoholischen Extraktes mit Natriumalkoholat verseift, dann in Wasser aufgenommen und mit Äther extrahiert. Der nach dem Verjagen des Äthers verbleibende Rückstand wurde in wenig heißem Alkohol gelöst und damit sowohl die Windaussche Digitoninprobe wie die Liebermannsche Reaktion angestellt. Beide fielen negativ aus.

Der andere Teil des alkoholischen Extrakts aus dem mit Baryumcarbonat eingedampften Filtrat wurde mit Säuregemisch verascht and dann mit Ammon. molybdat. nach Neumann auf Phosphorsäure geprüft. Auch diese Probe fiel negativ aus.

Durch die Phosphorwolframsäure werden also aus dem Blut Cholesterin wie Phosphatide quantitativ ausgefällt.

Des weiteren eignete sich das Filtrat außer zur Kohlenstoffbestimmung vorzüglich zur Ermittelung des Blutzuckers und des Gesamtacetons, es mußten sich ferner in ihm alle als Zuckerabbauprodukte in Frage kommenden Körper finden.

Mancini, der zuerst Restkohlenstoffbestimmungen im Blute, das mit Phosphorwolframsäure enteiweißt war, nach der von Spiro modifizierten Messingerschen Methode ausgeführt

1) Gemeint ist der gesamte Restkohlenstoff des enteiweißten Bluts.

2) Mancini, Bioch. Zeitschr., Bd. 26, 1910, S. 149. 
hat, berichtet über seine Ergebnisse in zwei Arbeiten. Zu der ersten $^{1}$ ) wurden vorwiegend Untersuchungen am Hundeblut und nur in drei Fällen an Menschenblut ausgeführt. In zweien davon handelte es sich um Placentarblut, in dem dritten um Blut einer Urämischen. Die Bestimmungen, die mit Serum ausgeführt waren, ergaben, auf $100 \mathrm{ccm}$ Serum berechnet, für das Placentarblut 173 und $179 \mathrm{mg}$, für das Urämieblut $214 \mathrm{mg}$ Kohlenstoff. Wir möchten hier gleich vorwegnehmen, daß unsere Werte im Gesamtblut sich auch etwa in dieser Höhe bewegen.

Die von Mancini in seiner zweiten Arbeit ${ }^{2}$ ) erhaltenen Befunde an Blut von Gesunden und Kranken ergaben andere Werte. Sie müssen hier außer Betracht bleiben, da sie unter Änderung der Methodik erhalten sind. Überdies hat Mancini Diabetikerblut nicht untersucht. ${ }^{3}$ )

Die von mir befolgte Technik schloß sich eng der von Mancini im Hofmeisterschen Laboratorium bei seiner ersten Arbeit geübten an. Auf besondere technische Einzelheiten, die mir bei der Veraschung des Kohlenstoffs auf nassem Wege beachtenswert erschienen, soll an anderer Stelle eingegangen werden.

1) Biochem. Zeitschr., Bd. 26, 1910, S. 149.

2) Biochm. Zeitschr., Bd. 32, 1911, S. 164.

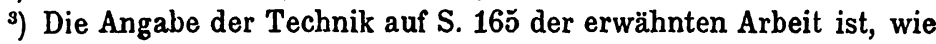
mir Herr Prof. Dr. Mancini auf meine Anfrage mitteilte, infolge Druckfehlers vollkommen unrichtig. Er arbeitete vielmehr folgendermaßen: Er ließ in einen $50 \mathrm{ccm} 10 \%$ iger Fluornatriumlösung enthaltenden Meßzylinder $50 \mathrm{ccm}$ Blut einfließen, fügte $100 \mathrm{ccm}$ Wasser zu, das $6 \mathrm{ccm}$ konzentrierte $\mathrm{H}_{2} \mathrm{SO}_{4}$ enthielt, und fällte mit $100 \mathrm{ccm} 10 \%$ iger Phosphorsäure. Soweit die persönlichen Angaben Mancinis. In der Arbeit heißt es über die Verarbeitung des Filtrats: •Das Ganze wurde filtriert und das ganze klare Filtrat in 2 gleiche Teile geteilt, die somit je $25 \mathrm{ccm}$ Blut entsprachen. In denselben wurde nach der früher beschriebenen Methode der Gehalt an Kohlenstoff durch Überführung in $\mathrm{CO}_{2}$ bestimmts. Wenn Herr Mancini so gearbeitet hat, wie es hier beschrieben ist, so muBte or viel zu niedrige Werte bekommen. Nach dem Filtrieren bleibt in dem feuchten, sehr voluminösen Niederschlag so viel Flüssigkeit zurück, daß das Gesamlfiltrat nur etwa 55-60\% des Gesamtvolumens nach der Fällung beträgt. Das Gesamtfiltrat entspricht daher durchaus nicht der verwandten Blutmenge und es ist folglich nicht gestattet, Werte, die aus der Hälfte des Filtrats erhalten sind, auf die Hälfte des Blutes umzurechnen. 
Im Verlaufe von ca. 200 Einzeluntersuchungen (stets Doppelbestimmungen) fand ich beim gesunden Menschen und bei Leichtkranken mit nur unwesentlichen, den Stoffwechsel unseres Wissens nicht beeinflussenden Störungen für $100 \mathrm{ccm}$ Gesamtblut Kohlenstoffwerte $\mathrm{zwisch} \in \mathbf{n} 170$ und $200 \mathrm{mg}$. Davon entfallen ca. $40 \mathrm{mg}$ auf den Kohlenstofi des Blutzuckers, es verbleiben also ca. 130-160 mg Kohlenstoff - Nichtzucker-Kohlenst off, - , die sich auf den Harnstoff, die Monaminosäuren, die Acetonkörper und unbekannte organische Verbindungen verteilen. Da der Harnstoff nur 20\% Kohlenstoff enthält und die Menge des Harnstoffs im Blute etwa $45-73 \mathrm{mg}$ beträgt, so treffen auf den Kohlenstoff des Harnstoffs nur etwa $9-15 \mathrm{mg}$, also eine recht kleine Menge, wenn man ihr die große Menge des auf unbekannte Körper entfallenden Kohlenstoffs gegenüberstellt.

Wie liegen nun die Verhältnisse beim Diabetes? Ist hier das Anwachsen des Blutzuckers von einem entsprechenden Anstieg des Kohlenstoffs begleitet? Denken wir uns einen Diabetesfall mit 0,3\% Blutzucker! Der Zuckerkohlenstoff würde hier $3 \cdot 40=120 \mathrm{mg}$ betragen und es würde sich, vorausgesetzt, daß die Menge des übrigen Kohlenstoffs gleich bliebe, ein Restkohlenstoff von $\left.120+145^{1}\right)=265 \mathrm{mg}$ ergeben. Und ein sehr hoher Blutzuckergehalt müßte zu ungeheuren Kohlenstoffzahlen führen, bei $0,9 \%$ würde der Restkohlenstoff einen Wert von $(9 \cdot 40)+145=505 \mathrm{mg}$ erreichen.

Wie liegen nun die Verhältnisse in Wirklichkeit? Ich habe bei einer Reihe von Diabetesfällen gleichzeitig den Restkohlenstoff und den Blutzucker bestimmt, bei verschiedenen wurden die Untersuchungen mehrmals wiederholt.

Ich ging so vor, daß ich das Blut meist frühmorgens dem noch nüchternen Patienten mit der Aderlaßnadel entnahm und es in einem mit pulverisiertem Natriumfluorid beschickten Meßzylinder auffing. Es wurden je nach Bedarf etwas mehr als 50 oder $100 \mathrm{ccm}$ entnommen. Dann wurde mit 10\% iger

1) 145 als Mittel des Nichtzuckerkohlenstoffs, der zwischen 130 und $160 \mathrm{mg}$ schwankt. 
Phosphorwolframsäure enteiweißt. Nach vielen Versuchen erschien mir das folgende Verfahren am raschesten und sichersten zum Ziele zu führen. In einem großen (etwa 500 oder $1000 \mathrm{ccm}$ ) Meßzylinder wurde zuerst das 4fache der zur Verarbeitung gelangenden Blutmenge an Wasser genau abgemessen (200 bezw. $400 \mathrm{ccm})$, das Doppelte an 10\% iger Phosphorwolframsäure (100 bezw. $200 \mathrm{ccm}$ ), dann auf je $100 \mathrm{ccm}$ Flüssigkeit je $2 \mathrm{ccm}$ konzentrierte Schwefelsäure (6 bezw. $12 \mathrm{ccm}$ ) zugegeben. Das Ganze wurde mit dem Glasstab gut durchgemischt, und nun aus der Pipette unter lebhaftem Rühren die gewählte Blutmenge ( 50 bezw. $100 \mathrm{ccm}$ ) langsam einlaufen gelassen. Dann wurde das Volumen notiert. Schon nach wenigen Minuten setzt sich der entstandene voluminöse Niederschlag $\mathrm{zu}$ Boden und man bekommt ein wasserklares Filtrat. In der Regel ließ ich nach der Fällung das Ganze einige (mindestens 3) Stunden stehen und filtrierte dann. Die weitere Verarbeitung erfolgte erst nach weiteren 12-24 Stunden. Nur in den seltensten Fällen trat beim Stehen des Filtrates noch ein leichter Niederschlag auf, der dann durch Filtrieren entfernt wurde. Für die Kohlenstoff bestimmung, die stets doppelt angesetzt wurde, gelangten in der Regel $50 \mathrm{ccm}$ des Filtrats zur Verwendung, weitere 50 bezw. 25 wurden zur Zuckerbestimmung nach Bertrand genommen (zuletzt auch hier stets Doppelbestimmungen!) Außerdem wurde regelmäßig der Stickstoff nach Kjeldahl und, wenn das Material reichte, das Gesamtaceton nach Schmitz-Embden bestimmt. Die Enteiweißung mit Phosphorwolframsäure eignet sich ganz vorzüglich zur Zuckerbestimmung im Gesamtblut. Nach ihrer Empfehlung durch Scheibler ${ }^{1}$ ) für die Zuckerisolierung aus eiweißhaltigen Lösungen hat sie Waymonth-Reid²) für die Blutzuckerbestimmung verwandt. Später hat sie Oppler ${ }^{3}$ ) wärmstens empfohlen. Er enteiweißte nicht wie Reid in der Wärme, sondern in der Kälte und entfernte nach dem Filtrieren die Phosphorwolframsäure mị Bleiacetat. Da es uns zunächst

1) Zit. nach Abderhalden, Handbuch der biochem. Arbeitsmethoden, Bd. 2, S. 51.

2) Ebenda, Bd. 5, S. 175.

3) Diese Zeitschr., Bd. 64, 1910, S. 393 und Bd. 75, 1911, S. 7. 
nur auf die Verwendung des Bertrandschen Verfahrens ankam, konnten wir das Filtrat ohne weitere Verarbeitung benutzen. Daß das Vorhandensein der Phosphorwolframsäure ${ }^{1}$ ) bei der Bertrandschen Zuckerbestimmung nicht stört, beweisen eigens angestellte vergleichende Zuckeranalysen in Blutplasma, in denen ich einmal mit Phosphorwolframsäure, das andere Mal mit kolloidalem Eisenhydroxyd nach Michaelis und Rona enteiweißte. Die Werte stimmten in der Regel auf den Tropfen $\mathrm{KMnO}_{\uparrow}$ genau überein.

In folgendem seien kurz die Ergebnisse der Untersuchungen zusammengestellt:

Fall I, Pat. Lu..., 33 Jahre alt, seit einigen Jahren Diabetes nachgewiesen, schwere Form.

1. Untersuchung am 16. V. 15, am Tage zuvor in $2100 \mathrm{ccm}$ Harn 10,5 g Zucker (polar. bestimmt) ausgeschieden.

Tagesmenge des Gesamtacetons $3,11 \mathrm{~g}$.

Blutzucker : $0,19 \%$.

$$
\begin{array}{cc}
\text { Rest-Kohlenstoff : } 222 \mathrm{mg} . \\
\text { Zucker- } \\
\text { Nichtzucker- }
\end{array}
$$

2. Untersuchung am 11. VI. 15, am Tage zuvor in $1600 \mathrm{ccm}$ Harn 19,2 g Zucker (polar.) ausgeschieden.

Tagesmenge des Gesamtacetons 1,28 g.

Blutzucker: $0,144 \%$. Gesamtaceton in 100 Blut: $11 \mathrm{mg}$.

Rest-Kohlenstoff : $206 \mathrm{mg}$.

$$
\begin{gathered}
\text { Zucker- } \quad \text { : } 57,6 \text {, } \\
\text { Nichtzucker- } \\
\text {, } 147,4 \text {, }
\end{gathered}
$$

3. Untersuchung am 1. XII. 15. Ausgebildetes Coma diabetic., 16 Stunden später $\dagger$.

Blutzucker: $0,5 \%$. Gesamtaceton in 100 Blut: $5,5 \mathrm{mg}$.

Rest-Kohlenstoff : 401,8 mg.

$\begin{array}{cl}\text { Zucker- } & \text { " } \\ \text { Nichtzucker- } & : 200 \text {, }\end{array}$

Fall II, Pat. Goi...., 27 Jahre alt, mittelschwere bis schwere Form.

1) Ich benutzte stets das Mercksche Präparat. 
Untersuchung am 8. X. 15, nüchtern. Am Tage zuvor (1. Hafertag) in $4100 \mathrm{ccm}$ Urin $82 \mathrm{~g}$ Zucker (polar.) ausgeschieden.

Tagesmenge des Gesamtacetons 12,5 g.

Blutzucker : 0,31\%. Gesamtaceton in 100 Blut $8,2 \dot{\mathrm{mg}}$. Rest-Kohlenstoff : $226 \mathrm{mg}$.

Zucker- \: 124 ,

Nichtzucker-. $\quad: 102$,

Fall III, Pat. Dr...., 55 Jahre alt, leichte Form.

Untersuchung am 8. X. 15 ambulatorisch; Pat. war 14 Tage vorher nach mehrwöchentlicher Behandlung entlassen worden; damals bei ca. 1000-1500 Urinmenge 5-10 g Zucker (polar.) in 24 Stunden ausgeschieden.

Blutzucker : $0,28 \%$. Gesamtaceton in 100 Blut $4,1 \mathrm{mg}$. Rest-Kohlenstoff : $214 \mathrm{mg}$.

$\begin{aligned} \text { Zucker- } & \text { * } \\ \text { Nichtzucker- } & \text { × } 112 \text {, }\end{aligned}$

Fall IV, Pat. Bü...., 42 Jahre alt, mittelschwere bis schwere Form.

1. Untersuchung am 12. X. 15, nüchtern. In den Tagen zuvor vorwiegend Gemüsediät, am 11. X. 153800 Urin mit 13,2 g Zucker (polar.).

$0,4 \%$ Blutzucker. Gesamtaceton in 100 Blut : 10,9 mg. Rest-Kohlenstoff : $213 \mathrm{mg}$.

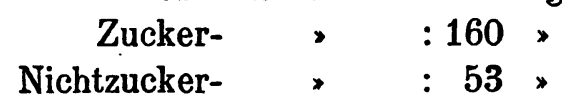

2. Untersuchung am 16. I. 16, nüchtern; am 15. I. 16 5500 Urin mit $110 \mathrm{~g}$ Zucker (polar.)

Tagesmenge des Gesamtacetons 3,54 g, während der letzten Tage nur Gemüse mit 100 Butter und Alkohol.

$0,44 \%$ Blutzucker.

Rest-Kohlenst off : $331,3 \mathrm{mg}$.

$\begin{array}{rll}\text { Zucker- } & : & : 176 \text { " } \\ \text { Nichtzucker- } & : & : 155,3 \text { " }\end{array}$

Fall V, Pat. Hi...., 33 Jahre alt, schwere Form.

Untersuchung am 14. XII. 15, nicht nüchtern. Am 13. XII. 15 in $3600 \mathrm{ccm}$ Urin 93,6 Zucker (polar.) ausgeschieden. Tagesmenge deś Gesamtacetons 1,17 g. 
In den letzten Tagen vorwiegend Gemüse +4 Eier $+80 \mathrm{~g}$ Butter.

$0,55 \%$ Blutzucker.

Rest-Kohlenstoff : $285,3 \mathrm{mg}$.

Zucker- , :220,

Nichtzucker- , : 65,3,

Fall VI, Pat. Kr...., 78 Jahre alt, leichte Form, Arteriosklerose, Emphysem.

Untersuchung am 27. XII. 15, nicht nüchtern. Am 26. XII. 15 in 2000 Urin $6 \mathrm{~g}$ Zucker (polar.) ausgeschieden, kein Aceton, keine Acetessigsäure.

Nahrung: gemischte Diät.

Blutzucker: $0,24 \%$.

$$
\begin{gathered}
\text { Rest-Kohlenstoff : } 209 \text { mg. } \\
\text { Zucker- }, \quad: 96, \\
\text { Nichtzucker- } \quad: 113,
\end{gathered}
$$

Fall VII, Pat. Se....., 18 Jahre alt.

Seit wenigen Wochen erst subj. Beschwerden (starker Durst, Hunger usw.), vorher nicht behandelt.

1. Untersuchung am 6. II. 16, nicht nüchtern, am Tage vorher in die Klinik aufgenommen, bisher gemischte Diät. Am Tage der Blutentnahme werden in 5800 Urin $340 \mathrm{~g}$ Zucker (polar.) ausgeschieden.

Blutzucker : $0,91 \% ;^{1}$ ) Gesamtaceton in 100 Blut: 10,4 mg.

Rest-Kohlenstoff : $353 \mathrm{mg}$.
Zucker-, 364,
Nichtzucker-,

2. Untersuchung am 11. II. 16.

Pat. hatte am 6. II. frei gewählte gemischte Diät, am 7. und 8. II. Gemüsetage, am 9. II. 1. Hafertag, am 10. II. 2. Hafer tag. An diesem Tage $2600 \mathrm{ccm}$ Urin, kein Zucker (Trommer negat.) kein Aceton.

Blutzucker: $0,445 \%$. Gesamtaceton in 100 Blut $1,2 \mathrm{mg}$.

1) Zucker im Plasma nach Enteiweißung mit Eisen: 0,98\%. 


\section{Rest-Kohlenstoff : $163 \mathrm{mg}$. \\ Zucker- , : 178 , \\ Nichtzucker-, : -}

3. Untersuchung am 7. III. 16.

In den letzten Wochen bei strenger Diat Harn stets völlig zuckerfrei (Trommer negativ), vom 29. II. ab strenge Diät und $25 \mathrm{~g}$ Brot, Harn zuckerfrei. Am 6. II. 16. Gemüsetag, 1500 Urin, Nachtrommer, Aceton negativ.

Blutzucker: $0,29 \%$.

Rest-Kohlenstoff : $254 \mathrm{mg}$.

Zucker- 》: 116 ,

Nichtzucker- , :145,

Fall VII, Pat. Sto....,, 26 Jahre alt, mittelschwerer Fall.

Untersuchung am 16. II. 16, nachmittags 5 Uhr; am 14. und 15. II. Gemüsetage; am 15. II. 16 in 1200 Urin 3,6 g Zucker (polarim.) ausgeschieden. Tagesmenge des Gesamtacetons $0,62 \mathrm{~g}$.

Blutzucker: $0,409 \%$.Gesamtaceton in 100 Blut $4,1 \mathrm{mg}$.

Rest-Kohlenstoff : $255,5 \mathrm{mg}$.

Zucker- »:163,6

Nichtzucker- $\quad: 91,9$ »

Ein kurzer Blick auf die Ergebnisse der Untersuchungen genügt, um sofort festzustellen:

In der überwiegenden Mehrzahl der Fälle ist der Rest-Kohlenst off wesentlich niedriger, als man nach dem Anwachsen des Blutzuckers erwarten sollte. Der Blutzucker nimmt also einen erheblich größeren Teil des Rest-Kohlenstoffes in Anspruch als in der Norm und der Nichtzucker-Kohlenst off wird kleiner.

Betrachten wir nun die Befunde im einzelnen!

Um möglichste Übersichtlichkeit zu gewinnen und besonders die Beziehungen von Zucker- zu Nichtzucker-Kohlenstoff deutlich zu machen, habe ich im folgenden die Verhältnisse graphisch dargestellt. Die Figuren sind ohne weiteres verständlich. Die Höhe der Rechtecke entspricht der Höhe der Kohlenstoffwerte und zwar zeigt $1 \mathrm{~mm}$ je $2 \mathrm{mg}$ Kohlenstoff an. In der Zusammenstellung sind sie nach der Höhe des Blutzuckers geordnet. 
Tabelle 1.

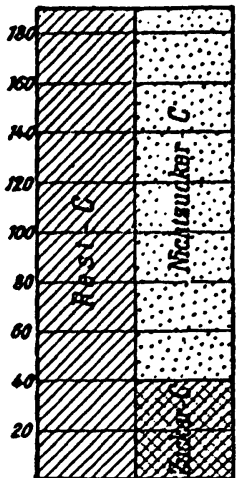

Normal.

Rest-C .. $=190 \mathrm{mg}$ Zucker-C. $=40$ ? (0,1\% Blutzucker) Nichtzucker-C $=150 \mathrm{mg}$

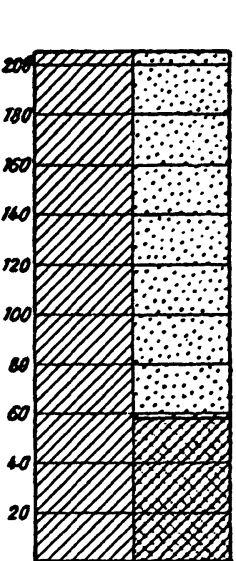

Fall I

(2. Untersuchung).

Rest-C . $=206 \mathrm{mg}$ Zncker-C $=57,6 \mathrm{mg}$ $(0,144 \%$ Blatzucker) Nichtzucker-C $=147,4 \mathrm{mg}$

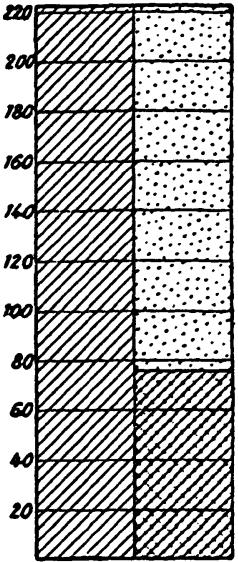

Fall I

(1. Untersuchung)

Rest-C . . = $222 \mathrm{mg}$ Zucker-C. $=76 \mathrm{mg}$ $(0,19 \%$ Blutzucker $)$ Nichtzucker-C $=146 \mathrm{mg}$

Tabelle 2.

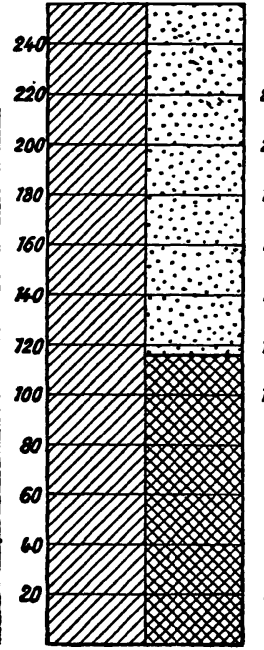

Fall VII

(3. Untersuchung)

Rest-C . . $=254 \mathrm{mg}$ Zucker-C $=116 \mathrm{mg}$ $(0,29 \%$ Blutzucker) Nicht-

zucker-C $=145 \mathrm{mg}$

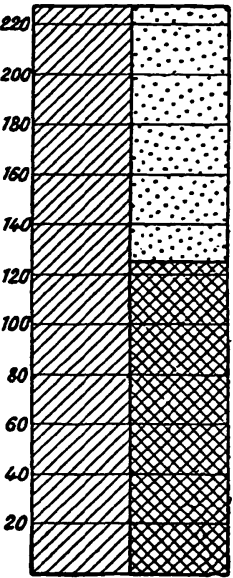

Fall II

Rest-C . . $=226 \mathrm{mg}$ Zucker-C. $=124 \mathrm{mo}$ $(0,31 \%$ Blutzucker) Nichtzucker-C = $102 \mathrm{mg}$ 
Tabelle 3.

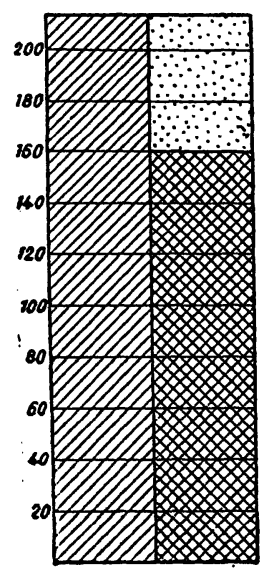

Fall IV

(1. Untersuchung)

Rest-C . . = $213 \mathrm{mg}$ Zucker-C. $=160 \mathrm{mg}$ $(0,4 \%$ Blutzucker)

Nicht-

zucker-C $=53 \mathrm{mg}$

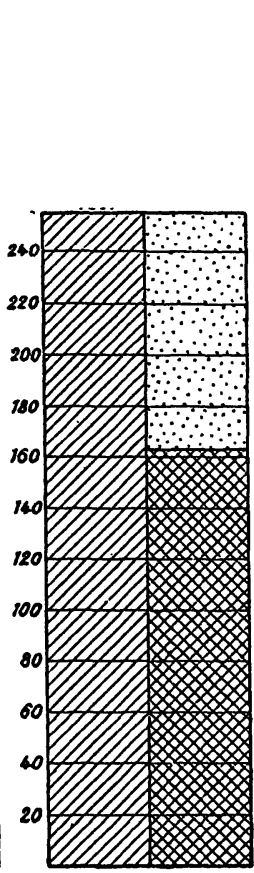

Fall VIII.

Rest-C . $=255,5 \mathrm{mg}$ Zucker- $\dot{\mathrm{C}}=163,6 \mathrm{mg}$ (0,409\% Blutzucker) Nicht-

zucker-C $=91,9 \mathrm{mg}$

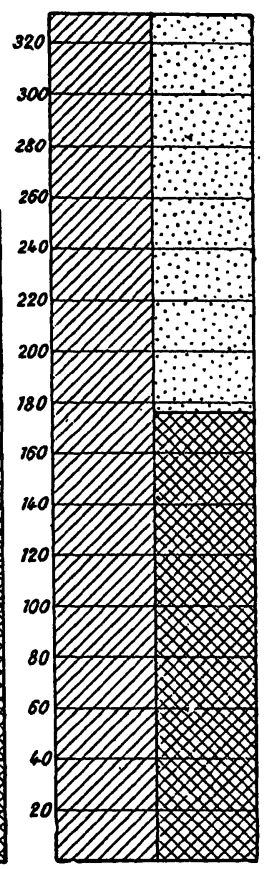

Fall IV

(2. Untersuchung)

Rest-C . = $331 \mathrm{mg}$ Zucker-C $=176 \mathrm{mg}$ $(0,44 \%$ Blutzucker) Nicht-

zucker-C $=155,3 \mathrm{mg}$

Im Normalblut macht die auf den Zucker entfallende Kohlenstoffmenge nur ungefähr $1 / \mathrm{s}$ des gesamten Restkohlenstoffs aus. Die Hauptmenge, der "Nichtzucker-Kohlensto ff », entfällt zum größten Teil auf unbekannte Verbindungen. Wir haben oben diese Verhältnisse schon kurz auseinandergesetzt. Beim Diabetes tritt - wenigstens bei der Mehrzahl der Fälle - eine ganz unerwartete Erscheinung auf: Der Restkohlenstoff wächst nicht in dem Maße, wie sich der BlutzuckerKohlenstoff vermehrt, m. a. W. der Zucker-Kohlenstoff nimmt zu, der Nichtzucker-Kohlenst off sinkt. So beträgt der Nichtzucker-Kohlenst off z. B. bei Fall III nur 102, bei Fall IV nur 53, bei Fall V $63 \mathrm{mg}$. Geradezu unverständlich erscheint auf den ersten Blick der Befund von Fall VII, Unter- 
Tabelle \&.

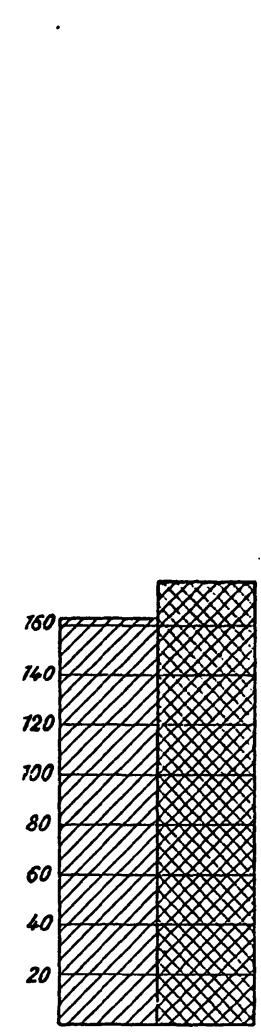

Fall VII

(2. Untersuchung)

Rest-C . . = $163 \mathrm{mg}$

Zucker-C $=178 \mathrm{mg}$

$(0,445 \%$

Nicht-

zucker-C $=\varnothing$

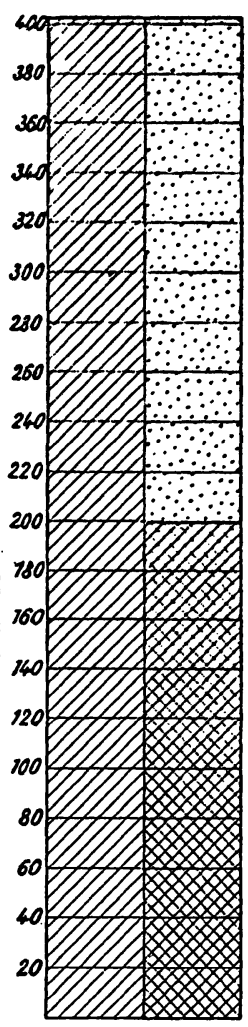

Fall I

(3. Untersuchung)

Rest-C . $=401,8 \mathrm{mg}$

Zucker- $\mathrm{C}=200 \mathrm{mg}$

Nicht-

zucker-C $=201,8 \mathrm{mg}$

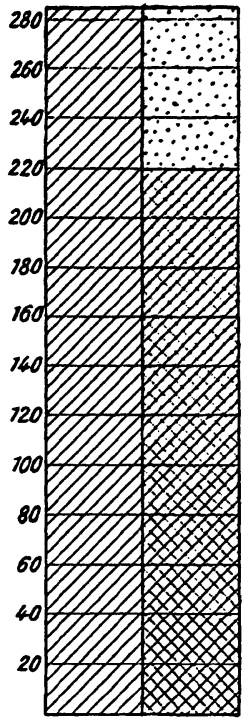

Fall V

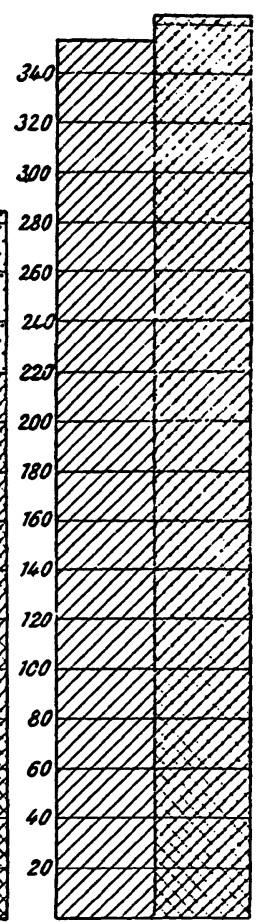

Fall VII

(1. Untersuchung)

Rest-C . $=285,3 \mathrm{mg}$ Rest $-C_{\text {. . }}=3553 \mathrm{mg}$ Zucker-C $=220 \mathrm{mg}$ Rucker-C $\cdot=36 \dot{\mathrm{mg}}$ Nicht $\quad(0,91 \%$ Blutzucker $)$ Nicht-

zucker-C $=\varnothing$

suchung 1 und 2. In der ersten Untersuchung hat hier die Blutzuckerbestimmung einen Wert von $0,91 \%$, d. h. $910 \mathrm{mg}$ Zucker in 100 Blut ergeben, die des Restkohlenstoffs $353 \mathrm{mg} \mathrm{C}$. In $910 \mathrm{mg}$ Glukose sind aber $364 \mathrm{mg}$ Kohlenstoff enthalten. Der Restkohlenstoff ist somit niedriger als der aus der Berechnung des Blutzuckers sich ergebende Zuckerkohlenst off. Es genügt also hier nicht einmal der Restkohlenstoff zur Deckung des Kohlenstoffwertes, der auf den Blutzucker allein entfallen muß. Eine zweite Untersuchung des 
Blutes bei demselben Patienten, der 5 Tage nach Ausscheidung einer Tagesmenge von $340 \mathrm{~g}$ Zucker im Urin keinen Zucker mehr aufwies, ergab den gleichen, zunächst unerklärlichen Befund. Auch diesmal war die Restkohlenstoffmenge kleiner als die dem Zuckergehalt entsprechende, im übrigen waren beide Werte etwa auf die Hälfte gesunken.

Wie kann man nun diese beiden höchst merkwürdigen Befunde deuten? Sie sollen zunächst besprochen werden.

Ohne weiteres ist das eine klar, daß der gesamte Restkohlenstoff unmöglich in seiner ganzen Menge auf den Blutzucker entfallen kann. Denn neben dem Blutzucker existiert in dem mit Phosphorwolframsäure enteiweißten Blut noch eine ganze Reihe von anderen kohlenstoffhaltigen Verbindungen. Beim Normalen macht ja der Zuckerkohlenstoff nur etwa $1 / \bar{j}$ des Gesamtkohlenstoffs aus: In erster Linie denken wir an die N-haltigen Körper. Daß sie in derselben Menge im Diabetikerblut vorhanden sind, wie im Blute des Gesunden, zeigt die Bestimmung des Stickstoffs, die wir stets neben der des Zuckers und des Kohlenstoffs durchgeführt haben. Wir haben in $100 \mathrm{ccm}$ Gesamtblut N-Werte zwischen 30 und $50 \mathrm{mg}$ gefunden. Es wurde bereits erwähnt, daß davon ungefähr $70 \%$ auf den Stickstoff des Harnstoffs entfallen, und daß somit der Harnstoffkohlenstoff auf etwa 9-15 mg zu schätzen wäre. Der Rest anderer stickstoffhaltiger Substanzen als Harnstoff beansprucht gleichfalls einige Milligramm Kohlenstoff. Sie lassen sich schwer abschätzen, da es eine große Zahl von stickstoffhaltigen Körpern gibt, wie z. B. gewisse Aminosäuren, die zu über $50 \%$ aus Kohlenstoff bestehen und bei denen auf $1 \mathrm{~N}$-Atom 6-9 C-Atome treffen. Doch das sind nur ganz grobe Abschätzungen. Jedenfalls bleibt von dem Nichtzucker-Kohlenstoff, auch wenn wir bekannte Verbindungen, wie die Acetonkörper, mit hohen Werten einsetzen, ein beträchtlicher Rest, von dem wir nicht wissen, welchen Verbindungen er angehört.

Wir kommen also nach diesen Überlegungen zu dem bindenden Schluß, daß in dem Falle VII (Untersuchung 1 und 2) die Blutzuckerwerte zu hoch sein müssen. An der Richtigkeit 
der Analysen an sich kann nicht gezweifelt werden. Alle Kohlenstoffbestimmungen sind Doppelbestimmungen, die gut übereinstimmen. Die Zuckeranalysen wurden zwar anfangs nicht stets, wie die Kohlenstoffbestimmungen, doppelt angesetzt, aber häufig durch eine Kontrolle auf ihre Richtigkeit geprüft. ${ }^{1}$ ) Analytische Fehler können also nicht vorliegen. Wir müssen nach einer anderen Erklärung suchen. Geben uns die verwandten Methoden die Gewähr, daß sie uns nun das anzeigen, was wir suchen? Der Kohlenstoff wird als Kohlensäure gewogen. Da kann eine Täuschung nicht vorliegen. Die Reaktion hingegen, die wir zur quantitativen Zuckerbestimmung verwenden, ist nicht nur der Glukose eigen, sondern einer ganzen Reihe von Substanzen, die die charakteristische Gruppe - $\mathrm{CH}$ OH. CObesitzen. Die reduzierende Kraft einer Lösung ist nun wahrscheinlich abhängig von der Zahl dieser Gruppen, sie sagt uns aber nichts darüber aus, ob an der reduzierenden Gruppe eine lange oder kurze Kohlenstoffkette hängt. Beim Traubenzucker kommt 1 Aldehydgruppe auf 6, beim Glycerinaldehyd z. B. nur auf 3 C-Atome. Es vermöchte also wahrscheinlich die Anwesenheit von Zuckern mit weniger als 6 C-Atomen den niedrigen Gesamtkohlenstoff zu erklären. $\left.{ }^{2}\right)$ Es würde dann der Zuckerkohlenstoff einen wesentlich niedrigeren Wert ergeben und die sparadoxe, Erscheinung verschwinden. Über die reduzierende $\mathrm{Kraft}$ des Glycerinaldehyds, ausgedrückt in Knpfer, konnte ich in der mir zugänglichen Literatur leider nichts finden.

Jedenfalls vermögen wir das eine mit aller Sicherheit zu sagen, daB in dem einen Fall neben Traubenzucker ein anderer reduzierender Körper mit entweder größerer Reduktionskraft oder niedrigerem Kohlenstoffgehalt als Traubenzucker im Blut vorhanden war.

Ist nun diese Auffassung auch bei den anderen Diabetesfällen mit den relativ zu niedrigen Kohlenstoffwerten geboten

1) Bei den späteren Untersuchungen wurden stets Doppelbestimmungen ausgeführt. Die Differenz betrug bei der Titration mit Kohle 0,4 , höchstens $0,1-0,15 \mathrm{ccm}$. In der Regel stimmten sie auf den Tropfen.

$\left.{ }^{2}\right)$ Die direkte Untersuchung auf Glycerinaldehyd mittels der × Kältereduktion, wurde nicht vorgenommen. 
oder kommen noch andere Erklärungsmöglichkeiten in Frage? Eine scheint mir sehr wohl der Erörterung wert. Nimmt man z. B. an, daß die in den Fällen III, IV (1. Untersuchung) und $\mathrm{V}$ erhaltenen Reduktionswerte nur auf Glukose zu beziehen sind, so würde hier in der Tat der Nichtzuckerkohlenstoff gegenüber der Norm stark vermindert sein. Diese Verminderung kann nicht die stickstoffhaltigen Kohlenstoffverbindungen betreffen, denn der Stickstoff hat den gleichen $\mathrm{W}^{\top}$ ert wie in der Norm. Es muß sich also um die stickstofffreien Kohlenstoffverbindungen handeln, über die wir nichts Näheres wissen. Vielleicht finden sich in dem Nichtzucker-Kohlenstoff des Gesunden Abbauprodukte des Zuckers, die Fehling sche Lösung nicht mehr reduzieren. Diese würden in unseren Diabetesfällen vermindert sein und man könnte $\mathrm{zu}$ folgender Vorstellung gelangen: Der Abbau des Zuckers ist gestört, es kommt zu einer Anhäufung des Zuckers und zu einer Verminderung seiner Abbauprodukte im Blut. Nehmen wir jedoch an, daß an den Reduktionswerten nicht nur Glukose, sondern noch andere Substanzen Anteil haben, so verlieren unsere quantitativen Überlegungen über die Verteilung des Kohlenstoffs auf zucker- und nichtzuckerartige Verbindungen an Sicherheit. Wir wissen nämlich nicht, wie große Mengen der reduzierenden Substanzen den gefundenen Kupferwerten entsprechen, und kommen über grobe Abschätzungen nicht hinaus. Ist so der Wert für die reduzierenden Körper unsicher, so vermögen wir die ihnen entsprechende Kohlenstoffgröße nicht mehr zu berechnen, auch wenn wir weiter ihren prozentischen Gehalt an Kohlenstoff wie beim Zucker mit $40 \%$ annehmen. Es wäre sehr verlockend, hier im Anschluß an die Arbeiten von Emb den und seinen Mitarbeitern ${ }^{1}$ ) an Körper wie den Glycerinaldehyd und das Dioxyaceton zu denken.

Wie man sieht, ist die Deutung unserer Befunde außerordentlich schwierig und gestattet eine ganze Reihe von Annahmen, ohne daß man einer davon einen besonderen Vorzug einräumen könnte.

1) Gustav Embden, Ernst Schmitz und Maria Wittenberg, Diese Zeitschr., Bd. 88, S. 210, 1913. Hierselbst finden sich Hinweise auf eine große Zahl von Arbeiten, die hierher gehören. 
oder kommen noch andere Erklärungsmöglichkeiten in Frage? Eine scheint mir sehr wohl der Erörterung wert. Nimmt man z. B. an, daß die in den Fällen III, IV (1. Untersuchung) und V erhaltenen Reduktionswerte nur auf Glukose zu beziehen sind, so würde hier in der Tat der Nichtzuckerkohlenstoff gegenüber der Norm stark vermindert sein. Diese Verminderung kann nicht die stickstoffhaltigen Kohlenstoffverbindungen betreffen, denn der Stickstoff hat den gleichen $\mathrm{W}^{\top}$ ert wie in der Norm. Es muß sich also um die stickstofffreien Kohlenstoffverbindungen handeln, über die wir nichts Näheres wissen. Vielleicht finden sich in dem Nichtzucker-Kohlenstoff des Gesunden Abbauprodukte des Zuckers, die Fehling sche Lösung nicht mehr reduzieren. Diese würden in unseren Diabetesfällen vermindert sein und man könnte zu folgender Vorstellung gelangen: Der Abbau des Zuckers ist gestört, es

\section{Druckfehlerberichtigung.}

Auf Seite 226, Fußnote 1, Zeile 2 Ende und Zeile 3 Anfang soll es heißen abei der Titration mit $\mathrm{KMnO}_{4}$ höchstens $0,1-0,15 \mathrm{ccm}$ * (statt bei der Titration mit kohle $0,4 \ldots$ ) 
Außer den Diabetesfällen mit relativ niedrigen Kohlenstoffwerten haben wir auch solche, bei denen sich der Gesamtkohlenstoff um soviel erhöht, als man nach dem Zuckerzuwachs von vorneherein erwartet hätte. Besonders hoch ist er in dem Fall von Coma diabeticum. Hier wird der hohe Wert wohl - wenigstens zum Teil - auf die Acetonkörper

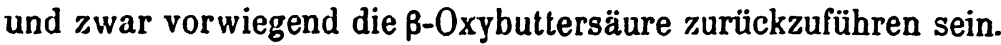
Das Gesamtaceton ist geringer als bei anderen Diabetesfällen. Die Frage übrigens, ob beim Diabetes außer Zucker noch andere reduzierende Substanzen im Blute kreisen, ist vielfach untersucht worden, ohne daß sich übereinstimmende Ergebnisse hätten erzielen lassen. Während eine Reihe von Autoren, wie Lyttkens und Sandgren, ${ }^{1}$ Schumm und Hegler ${ }^{2}$ ) und Paul Mayer ${ }^{3}$ ) nach vollkommener Vergärung des Serums bezw. des Vollbluts noch reduzierende Substanzen nachweisen konnten, fanden Frank und Bretschneider ${ }^{4}$ ) im menschlichen Blut keinerlei Restreduktion. Ebenso ablehnend äußerten sich Griesbach und Straßner. ${ }^{5}$ ) Neuerdings hat 0. Schumm $^{6}$ ) in umfangreichen Untersuchungen das Problem der Restreduktion neu bearbeitet. Er kommt zu dem Ergebhis, daB die Anschauung Griesbachs und Straßners, wonach die Reduktions- und Polarisationswerte praktisch durch die vorhandene Menge Traubenzucker bestimmt wurden, nicht zu Recht besteht. Es gelang ihm, mit aller Sicherheit das Vorhandensein von reduzierenden Substanzen in vergorenen Blutauszügen nachzuweisen. Damit findet die ältere Anschauung von dem Bestehen einer Restreduktion eine neue Stütze. Wie groß der Umfang der Restreduktion beim Diabetes ist, wissen wir noch nicht mit Sicherheit. Nach unseren Befunden könnte man vielleicht für manche Diabetesfälle eine recht erhebliche Restreduktion erwarten. Man muß sich hierbei jedoch gegenwärtig

1) Biochem. Zeitṣchr., Bd. 31, S. 153, 1911.

2) Zit. nach Schumm, Diese Zeitschr., Bd. 96, Heft 3, S. 204, 1915.

3) Biochem. Zeitschrift, Bd. 50, S. 362, 1913.

4) Diese Zeitschr., Bd. 71, S. 157, 1911; Bd. 76, S. 233, 1912.

5) Ebenda, Bd. 88, S. 199, 1913.

6) Ebenda, Bd. 96, S. 204, 1915. 
halten, daß manche niedere Zucker von Hefe vorzüglich vergoren werden. So fanden E. Buchner und J. Meisenheimer, $\left.{ }^{1}\right)$ daß die Gärfähigkeit des Dioxyacetons in einigen ihrer Versuche «direkt der des Traubenzuckers gleichkam», während der Glycerinaldehyd nur sehr langsam und unvollständig vergoren wurde. Ferner ist zu bedenken, daß es nach Neuberg und Ishida ${ }^{2}$ ) eine «zuckerfreie Hefegärung» gibt.

Die Kohlenstoff bestimmung im enteiweißten Blut gestattet, wie man sieht, überraschende Einblicke in den Kohlenhydratstoffwechsel. Sie zeigt, daß die Verhältnisse im Blute des Diabetikers nicht einfach in der Weise eine Änderung erfahren, daB der Blutzucker sich vermehrt, während sonst im Blut alles unverändert bleibt. ${ }^{3}$ ) Es treten vielmehr beim Diabetes große Umwälzungen im Gebiet der Kohlenhydrate auf. Aber wir sehen auch, daß zwischen den einzelnen Diabetesfällen große Differenzen bestehen, ja sogar bei dem einzelnen Fall, wenn man zu verschiedenen Zeiten untersucht. Es erscheint nicht unmöglich, auf diese Weise zu einer besonderen Gruppierung innerhalb des Diabetes zu gelangen. Wir halten es jedoch für verfrüht, jetzt schon bestimmtere Anschauungen auszusprechen.

Endlich hat die Kohlenstoff bestimmung im Blute gezeigt, daß es nicht angängig ist, die bei der Blutzuckerbestimmung mit Hilfe von Reduktionsmethoden erhaltenen Werte ohne weiteres auf Zucker umzurechnen. In gewissen Diabetesfällen sind sicher neben Zucker erhebliche Mengen von anderen reduzierenden Substanzen vorhanden.

\section{Zusammenfassung.}

1. Im Blut von Diabetikern, das mit Phosphorwolframsäure enteiweißt ist, wird der sogenannte Restkohlenstoff bestimmt, um so bei gleichzeitiger Blutzuckerbestimmung einen Überblick über die Verteilung des Kohlenstoffs,auf zucker- und nichtzuckerartige Substanzen zu erhalten.

2. Dabei ergibt sich die überraschende Tatsache, daß in der Mehrzahl der Fälle der Restkohlenstoff nicht in dem Maße

1) Ber. d. Deutsch. Chem. Ges., Bd. 43, S. 1773 (1910).

2) Biochem. Zeitschr., Bd. 37, S. 142, 1911.

3) Von den Acetonkörpern sei hier abgesehen. 
in die Höhe geht, wie man nach der Erhöhung des Blutzuckers erwarten würde. Rechnet man die dem Blutzucker entsprechende Kohlenstoffmenge aus, so entfällt die Hauptmenge des gesamten Restkohlenstoffs auf Zucker und es verbleibt nur eine kleine Menge von sNichtzucker-Kohlenstoff .

Das Verhältnis von \&Zucker- $>$ zu \&Nichtzucker-Kohlenstoff, ist also ganz anders als in der Norm.

3. In einem Fall (bei zwei zeitlich auseinander liegenden Untersuchungen) wird ein auf den ersten Blick völlig unverständlicher Befund erhoben: Der Zuckerkohlenstoff ergibt einen höheren Wert als der gesarnte Restkohlenstoff. Ein methodischer Fehler kann nicht vorliegen, es gibt nur eine Erklärung: Die mittels der Bertrandschen Methode erhaltenen Reduktionswerte können unmöglich allein auf Glukose bezogen werden. Es müssen außer Glukose noch eine oder mehrere andere reduzierende Substanzen vorhanden sein, die entweder ein stärkeres Reduktionsvermögen oder einen niedrigeren Kohlenstoffgehalt haben als Glukose.

4. Man wird daher in Zukunft die aus Blut durch Reduktionsmethoden gewonnenen Werte nicht mehr ohne weiteres als Zucker rechnen dürfen.

Anmerkung. Nach Abschluß der vorstehenden Arbeit konnte ich bei einem weiteren Diabetesfall im Blut einen schon in der Kälte reduzierenden Körper nachweisen. Die Reduktion betrug ca. 1/s der Gesamtreduktion in der Wärme. Es wird sich empfehlen, in Zukunft mittels der «Kältereduktion > auf niedrige Zucker im Blute zu fahnden.

Anhang.

Analytische Belege.

Fall I.

1. Untersuchung. $50 \mathrm{ccm}$ Filtrat (entspr. 6,972 Blut) ergeben

1. $0,0575 \mathrm{~g} \mathrm{CO}_{2}$

2. $\frac{0,0571, \mathrm{CO}_{2}}{0,0573 \mathrm{~g} \mathrm{CO}}$

Für 100 Blut $0,811 \mathrm{~g} \mathrm{CO}_{2}$ und $0,222 \mathrm{~g} \mathrm{C}$.

2. Untersuchung. $50 \mathrm{ccm}$ Filtrat (entpr. $7,045 \mathrm{Blut}$ ) ergeben

1. $0,0527 \mathrm{~g} \mathrm{CO}_{2}$

2. $0,0539>\mathrm{CO}_{2}$

Mittel $0,0 \tilde{5} 33 \mathrm{~g} \mathrm{CO}_{2}$. 
Für 100 Blut $0,756 \mathrm{~g} \mathrm{CO}_{2}$ und $0,206 \mathrm{~g} \mathrm{C}$.

3. Untersuchung. $50 \mathrm{ccm}$ Filtrat (entspr. 7,045 Blut) ergeben

1. $0,1046 \mathrm{~g} \mathrm{CO}_{\mathrm{z}}$

$$
\text { 2. } \frac{0,1031, \mathrm{CO}_{2}}{0,1038, \mathrm{CO}_{2}} \text {. }
$$

Für 100 Blut 1,507 $\mathrm{g} \mathrm{CO}_{2}$ und $0,4018 \mathrm{~g} \mathrm{C}$.

\section{Fall II.}

$50 \mathrm{ccm}$ Filtrat (entspr. 7,045 Blut) ergeben

$$
\begin{aligned}
\text { 1. } & 0,0591 \mathrm{~g} \mathrm{CO}_{2} \\
\text { 2. } & 0,0576>\mathrm{CO}_{2} \\
\text { Mittel } & 0,0583 \mathrm{~g} \mathrm{CO}_{2}
\end{aligned}
$$

Für 100 Blut $0,827 \mathrm{~g} \mathrm{CO}_{8}$ und $0,226 \mathrm{~g} \mathrm{C}$.

\section{Fall III.}

$50 \mathrm{ccm}$ Filtrat (entspr. 7,045 Blut) ergeben

$$
\begin{aligned}
& \text { 1. } 0,0551 \mathrm{~g} \mathrm{CO} \mathrm{CO}_{2} \\
& \text { 2. } 0,0559, \mathrm{CO}_{3}
\end{aligned}
$$

Für 100 Blut $0,787 \mathrm{~g} \mathrm{CO}_{2}$ und $0,214 \mathrm{~g} \mathrm{G}$.

Fall IV.

1. Untersuchung. $50 \mathrm{ccm}$ Filtrat (entspr. 7,045 Blut) ergeben

1. $0,0555 \mathrm{~g} \mathrm{CO}_{2}$

2. $0,0547, \mathrm{CO}_{2}$

Mittel $\overline{0,0551>\mathrm{CO}_{2}}$.

Für 100 Blut $0,082 \mathrm{~g} \mathrm{CO}_{2}$ und $0,213 \mathrm{~g} \mathrm{C}$.

2. Untersuchung. $25 \mathrm{ccm}$ Filtrat (entspr. 3,5225) Blut ergeben

1. $0,0444 \mathrm{~g} \mathrm{CO}_{2}$

$$
\text { 2. } \frac{0,0413>\mathrm{CO}_{2}}{0,0428 \mathrm{~g} \mathrm{CO}_{2}} \text {. }
$$

Für 100 Blut $1,215 \mathrm{~g} \mathrm{CO}_{2}$ und $0,3313 \mathrm{~g} \mathrm{G}$.

\section{Fall $\mathbf{V}$.}

$50 \mathrm{ccm}$ Filtrat (entspr. 7,045 Blut) ergeben

$$
\begin{aligned}
\text { 1. } 0,0706 \mathrm{~g} \mathrm{CO}_{2} \\
\text { 2. } \\
0,0768>\mathrm{CO}_{2} \\
0,0737 \mathrm{~g} \mathrm{CO}_{2}
\end{aligned}
$$

Für 100 Blut $1,095 \mathrm{~g} \mathrm{CO}_{2}$ und $0,2853 \mathrm{~g} \mathrm{C}$.

\section{Fall VI.}

$50 \mathrm{ccm}$ Filtrat (entspr. 7,045 Blut) ergeben

$$
\begin{aligned}
\text { 1. } & 0,0536 \mathrm{~g} \mathrm{CO}_{2} \\
\text { 2. } & 0,0543>\mathrm{CO}_{2} \\
\text { Mittel } & 0,0540 \mathrm{~g} \mathrm{CO}_{2}
\end{aligned}
$$

Für 100 Blut $0,7683 \mathrm{~g} \mathrm{CO}_{2}$ and $0,209 \mathrm{~g} \mathrm{C}$. 
232 W. Stepp, Zur Kenntnis des Zuckerabbaus beim Diabetes mellitus.

\section{Fall VII.}

1. Untersuchung. $50 \mathrm{ccm}$ Filtrat (entspr. $5,449 \mathrm{Blut}$ ) ergeben

1. $0,0695 \mathrm{~g} \mathrm{CO}_{2}$

2. $\frac{0,0716}{0,0706}=\frac{\mathrm{CO}_{2}}{\mathrm{CO}_{2}}$.

Für 100 Blut $1,2956 \mathrm{~g} \mathrm{CO}_{2}$ und $0,353 \mathrm{~g} \mathrm{C}$.

2. Untersuchung. $50 \mathrm{ccr}$ Filtrat (entspr. 7,045 Blat) ergeben

1. $0,0417 \mathrm{~g} \mathrm{CO}_{2}$

2. $\frac{0,0425, \mathrm{CO}_{2}}{0,0421, \mathrm{CO}_{2}}$

Für 100 Blut $0,5976 \mathrm{~g} \mathrm{CO}_{2}$ and $0,163 \mathrm{~g} \mathrm{C}$.

3. Untersuchung. $50 \mathrm{ccm}$ Filtrat (entspr. 7,045 Blat) ergeben

1. $0,0650 \mathrm{~g} \mathrm{CO}_{2}$

2. $\frac{0,0664, \mathrm{CO}_{2}}{0,0657 \mathrm{~g} \mathrm{CO}_{2}}$.

Für 100 Blut $0,932 \mathrm{~g} \mathrm{CO}_{2}$ and $0,254 \mathrm{~g} \mathrm{C}$.

\section{Fall VIII.}

$50 \mathrm{ccm}$ Filtrat (entspr. 7,045 Blut) ergeben

1. $0,0651 \mathrm{~g} \mathrm{CO}_{2}$

2. $\frac{0,0669=\mathrm{CO}_{2}}{0,0660 \mathrm{~g} \mathrm{CO}_{2}}$.

Für 100 Blut $0,9368 \mathrm{~g} \mathrm{CO}_{2}$ und $0,2555 \mathrm{~g} \mathrm{C}$. 